\title{
La definitud en chino: una breve comparación entre español y chino ${ }^{1}$
}

DOI: $10.32870 /$ mycp.v3i8.465

Cao Yufei ${ }^{2}$

\section{Resumen}

En este trabajo se analizan los instrumentos lingüísticos que codifican la definitud en chino, una lengua que no cuenta con artículos, y se establece una comparación entre español y chino de acuerdo con el modelo del estudio contrastivo. Aparte de las perspectivas léxica, morfológica y posicional, tomamos una visión textual para estudiar la definitud en la lengua china.

Palabras clave: definitud, artículo definido, clasificador numeral, comparación entre español y chino.

\section{DEFINITENESS IN CHINESE: A BRIEF COMPARISON BETWEEN SPANISH AND CHINESE}

\section{Abstract}

This paper analyzes the linguistic instruments that encode definiteness in Chinese, a language which has no articles. The paper also makes a comparison between Spanish and Chinese based on the contrastive study model. Besides

1. Este trabajo ha sido financiado por la Fundación de Investigaciones Científicas para los profesores de la Universidad de Estudios Internacionales de Shanghai (上海外国语大学校级一般科研项 目“语际距离理论框架下的汉西对比研究及应用”阶段成果).

Artículo recibido el 13 de enero de 2014 y dictaminado el 22 de febrero de 2014.

2. Universidad de Estudios Internacionales de Shanghai. Correo electrónico: yufeielisa@qq.com 
the lexical, morphological and positional perspectives, we hold a textual vision to study the definiteness in Chinese.

Keywords: definiteness, definite article, numeral classifier, comparison between Spanish and Chinese.

\section{Introducción}

Este trabajo se basa en el modelo del estudio contrastivo que propone Xu (1992: 14). Según este investigador, todos los idiomas tienen sus propios recursos lingüísticos para cumplir una determinada función $\mathrm{X}$. Al hacer la comparación de las dos lenguas $\mathrm{A}$ y $\mathrm{B},{ }^{3}$ tenemos que fijarnos en los diferentes recursos $(\mathrm{Xa}$ y $\mathrm{Xb})$ que se utilizan para llevar a cabo la misma función $\mathrm{X}$ :

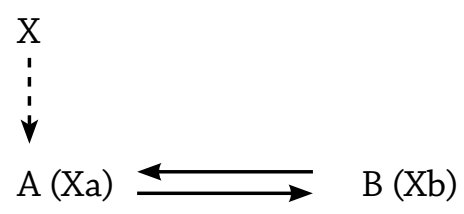

Al comparar el español con el chino, nos llama mucho la atención el hecho de que en los textos en español las expresiones con artículo definido aparezcan con una frecuencia muy elevada, mientras que en chino no hay artículos. ¿Por qué la ausencia del artículo en chino no impide la codificación y la identificación de los referentes? Y ¿cuáles son los recursos lingüísticos a que acude la lengua china a la hora de expresar la definitud? Éstas son las preguntas de partida de nuestro estudio y constituyen al mismo tiempo el objetivo de la investigación. En este trabajo haremos primero una comparación entre español y chino desde la perspectiva de definitud (X, según el modelo contrastivo) y analizaremos después el mecanismo en chino ( $\mathrm{Xb}$, según el modelo) que contribuye a la construcción de los referentes definidos.

3. En nuestro caso, A: español; B: chino. 


\section{Definitud e indefinitud: comparación entre español y chino}

En español, los sintagmas nominales (SSNN) definidos son marcados por el artículo definido. De acuerdo con RAE (2010: 268), los grupos nominales que llevan artículo definido son definidos, puesto que denotan entidades que el hablante supone identificables en un contexto a partir del contenido léxico del sustantivo y de la información que comparte con su interlocutor, denominada información consabida. Hay que señalar que los conceptos de definitud vs. indefinitud deben distinguirse de los de especificidad vs. inespecificidad. Es decir, los SSNN definidos pueden ser inespecíficos y también existen casos en que los SSNN indefinidos son específicos. Veamos algunos ejemplos de RAE (2010: 264):
a. Todavía no ha nacido la persona que pueda hacerla feliz.
b. El ganador obtendrá un auto como premio.
c. Tengo un vecino muy molesto.

En (1a), el SN definido no alude a una persona en particular, sino a un individuo hipotético que ha de cumplir ciertas condiciones. De manera análoga, el sujeto de la oración (1b) podría designar a una persona inexistente. Estos SSNN son definidos porque denotan entidades identificables y únicas, pero a la vez son inespecíficos. En cambio, el grupo subrayado en (1c) es indefinido, pero a la vez específico, puesto que se refiere a un individuo particular.

Como hemos explicado que la definitud no equivale a la especificidad, entonces ¿qué es definitud? ¿Cómo la podemos definir? Lyons nos propone una definición bastante interesante utilizando el término de "gramaticalización”. Según apunta este investigador (Lyons, 1999: 278), la definitud es la gramaticalización de los conceptos semántica o pragmáticamente definidos. Cabe mencionar que el término "gramaticalización" en la definición de Lyons se está utilizando en un sentido sincrónico (no diacrónico).
Al comparar el español con el chino, nos llama mucho la atención el hecho de que en los textos en español las expresiones con artículo definido aparezcan con una frecuencia muy elevada, mientras que en chino no hay artículos 
En español, con el artículo definido los conceptos semántica o pragmáticamente definidos son marcados y representados gramaticalmente. En la lengua china no hay artículos, es decir, en esta lengua los conceptos semántica o pragmáticamente definidos no son marcados. En palabras de Lyons (1999: 277), igual que otras categorías gramaticales, la definitud es una categoría gramatical que no se presenta necesariamente en todas las lenguas. Por ejemplo, en la lengua china no existe esta categoría. Sin embargo, hay que tener en cuenta que a pesar de la ausencia de la representación gramaticalizada de los conceptos definidos en chino, el concepto de definitud sí existe en esta lengua. Siguiendo este modelo de análisis, es decir, con la suposición de que la ausencia de la categoría del artículo definido en chino implica la no gramaticalización del concepto de definitud, entonces ¿qué implicaría la ausencia de la categoría de los clasificadores numerales (CLF.Num) en español?

En español no hay CLF.Num, mientras que en chino no existe el artículo. Este hecho nos hace pensar que estos dos elementos lingüísticos, que son aparentemente tan diferentes, deben ser mutuamente complementarios. Dicho de otro modo, viendo la pregunta arriba planteada desde el otro lado, podemos proponer que la ausencia de los CLF.Num en español implica la no gramaticalización de los conceptos indefinidos. Es decir, mediante los CLF. Num del chino, los conceptos semántica o pragmáticamente indefinidos son marcados y representados gramaticalmente. En chino, cuando un CLF.Num se utiliza independientemente, es decir, sin combinación con los numerales o demostrativos ni en su uso repetitivo, expresa la indefinitud. Por ejemplo: ${ }^{4}$

$$
\begin{aligned}
& \text { 他是个独自在湾流中的一条小船上钧鱼的老人, 至今已去了八十四 } \\
& \text { 天, 一条鱼也没逮住。头四十天里, 有个(CLF.Num) 男孩子(N)跟他在 } \\
& \text { 一起。可是, [...] }
\end{aligned}
$$

"Era un viejo que pescaba solo en un bote en la corriente del Golfo y hacía ochenta y cuatro días que no cogía un pez. En los primeros cuarenta días había tenido consigo a un muchacho."

En (2), el grupo nominal subrayado en chino, que está compuesto por un CLF.Num 个/ge/ y un nombre 男孩子/nan hai zi/ “muchacho", tiene una interpretación indefinida "un muchacho". La afirmación de que en español la definitud está gramaticalizada mientras que en chino lo gramaticalizado es la indefinitud, es bastante original e interesante. Con esta afirmación queremos

4. Ejemplo extraído de El viejo y el mar. 
resaltar que lo que realmente interesa a las lenguas son las oposiciones lingüísticas y no las maneras de marcarlas. Más concretamente, en la oposición de definitud $v s$. indefinitud, una lengua puede optar por marcar la definitud o decide tomar la otra opción: marcar la indefinitud. La primera opción la toma el español, mientras que la última la adopta el chino. Es algo parecido a que la misma realidad puede ser descrita desde dos perspectivas opuestas, ya que "el vaso está medio lleno" y "el vaso está medio vacío" transmiten el mismo mensaje. A continuación desarrollaremos nuestras ideas con más argumentos. Los dos puntos que vamos a desarrollar son:

a. hay + CLF.Num $+\mathrm{N}$

b. indicación del sujeto $v s$. indicación del complemento

En primer lugar, se observa que en chino, después del verbo 有/you/ "hay", que suele imponer a su complemento limitaciones relativas a su naturaleza indefinida, el CLF.Num siempre se usa solo, sin combinarse con el numeral e indica al mismo tiempo la indefinitud. Por ejemplo:
a. 桌上(en la mesa)有(hay)本(CLF.Num singular)书(libro)。
"Hay un libro en la mesa."
b. 桌上(en la mesa)有(hay)些(CLF.Num plural)水果(fruta)。
"Hay frutas en la mesa."

En (4), con la omisión del numeral uno después del verbo hay, se debilita la función original de los CLF.Num como mediación de un numeral y un sustantivo. Lo más importante que expresan los CLF.Num en estas oraciones es la indefinitud. Es decir, con los CLF.Num la indefinitud está representada gramaticalmente.

En segundo lugar, durante la evolución diacrónica, al desaparecer la flexión nominal, el sustantivo en español sólo expresa el significado y las relaciones sintagmáticas se encomiendan a elementos adjuntos. Por ejemplo, en latín, pater en nominativo tanto indica "padre" como "sujeto de frases" (Alonso, 1967). Debido a la ausencia de la flexión nominal, el español moderno acude al artículo definido para la indicación del sujeto. En otras palabras, pater del latín en nominativo toma en el español moderno la forma "el padre".

En chino se registra una evolución parecida. Con la desaparición del significado de cantidad, el sintagma "uno +CLF.Num" comenzó a tomar la forma 
“(uno)+个/ge/" o “个/ge/", 5 y su uso como señal de un complemento se hacía cada día más frecuente. Además, como el chino es una lengua analítica que se caracteriza por la carencia de la flexión, para señalar la función sintáctica de complemento que desempeñan unas expresiones nominales la gente empezó a utilizar el "CLF.Num general" en combinación con los verbos. En la dinastía Beisong ${ }^{6}$ ya se registraron casos de este uso, por ejemplo: ${ }^{7}$

$$
\begin{aligned}
& \text {...将知尔行脚, 驴年得个休歇 (lograr + “ge” + descansar: lograr un } \\
& \text { descanso)么! (道原, 《景德传灯录》) }
\end{aligned}
$$

Más tarde el “个/ge/” comenzó a aplicarse no sólo a los verbos y a los adjetivos sino también a las frases verbales, e incluso oraciones subordinadas que sirven como complemento en una oración:

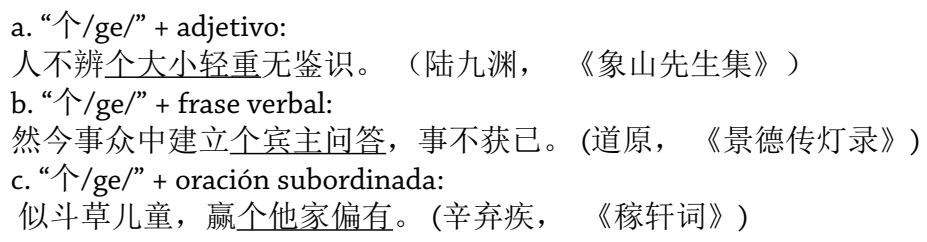

Además, cabe señalar que en chino la omisión del numeral es frecuente con los complementos pero no es posible con los sujetos. Este hecho no es difícil de explicar, puesto que siendo una representación gramaticalizada de indefinitud, el CLF.Num solo no debe aparecer en los sujetos, que requieren en la mayoría de los casos una interpretación definida.

Queremos resaltar que en los ejemplos (6a-c) todos los CLF.Num están empleados de manera aislada, es decir, no están en combinación con los numerales. Este tipo de CLF.Num son los más representativos de la gramaticalización, ya que según Heine (1997: 76), cuanto más camino recorre un elemento desde un numeral hasta una representación de indefinitud, más afectado será por los fenómenos del proceso de gramaticalización, como el blanqueo, la cliticización y la erosión fonética, etc. Al desaparecer el numeral en el sintagma "uno - /yi/ + CLF.Num", se blanquea la indicación de la cantidad y al mismo tiempo se produce la erosión fonética de "yi". En la última fase

5. 个/ge/: CLF.Num general.

6. Dinastía Beisong: 北宋960-1127.

7. Ejemplos de Zhang (2001). 
de tal proceso, el CLF.Num solo se vuelve cliticizado y gramaticalizado como representación de la indefinitud.

Los argumentos y ejemplos arriba expuestos apoyan la observación que ha hecho Chen (2004: 1177): "en chino no existen marcas simples y completamente gramaticalizadas de definitud. La definitud como una categoría gramatical aún no se ha desarrollado en la lengua china."

\footnotetext{
"There is no simple, fully grammaticalized marker of definiteness in Chinese, like the definite article in English [...] This leads to the conclusion that definiteness as a grammatical category, as defined in the narrow sense of the term, has not been fully developed in Chinese."
}

En resumen, diacrónicamente el artículo definido del español empieza a ser la señal del sujeto, mientras que el CLF.Num del chino empieza a ser la indicación del complemento. Sincrónicamente, la definitud en español está gramaticalizada, mientras que en chino la indefinitud está representada gramaticalmente. Dong (2010) sostiene la misma opinión:

“一+量”可以看作不定指的标记。”

“La estructura 'uno + CLF.Num' puede ser considerada como marca de indefinitud."

Todo esto muestra que el artículo definido del español y el CLF.Num del chino siempre marcan las mismas oposiciones lingüísticas de modos contrarios. Este hecho puede explicar en cierto modo la razón por la que en chino la definitud sigue sin estar marcada, puesto que con la indefinitud marcada, es de esperar que sea innecesaria la gramaticalización completa de la definitud.

\section{La definitud en chino a nivel subtextual}

Hemos llegado a la conclusión parcial de que en chino la definitud no está plenamente gramaticalizada. Sin embargo, tal conclusión no implica la inexistencia del concepto de definitud en esta lengua. Las preguntas que quedan por efectuar son: ¿cuáles son los grupos nominales que se consideran definidos en chino?

Chen (2004: 1151) propone tres tipos de instrumentos para expresar la definitud en chino, a saber, léxico, morfológico y posicional. Resumamos los tres instrumentos para expresar la definitud en lo que sigue: 

a. léxico: demostrativos, posesivos y cuantificadores universales
b. morfológico: repetición del CLF.Num
c. posicional

No prestaremos mucha atención al instrumento léxico, puesto que en español los grupos nominales modificados por los demostrativos, posesivos y cuantificadores universales también son definidos. En otras palabras, no se nota en este instrumento la singularidad de la lengua china. A continuación veamos el segundo instrumento: la repetición del CLF.Num. En chino este tipo de repetición se traduce como cuantificador universal anafórico de plural en español; por ejemplo:

$$
\begin{aligned}
& \text { 安娜的孩子(hijo de Ana) 个个( CLF.Num+CLF.Num) 机灵(listo)。 } \\
& \text { “Todos los hijos de Ana son listos." }
\end{aligned}
$$

En este ejemplo la instrucción de la repetición del CLF.Num 个/ge/ para el lector consiste en que a éste se le indica que no realice ninguna selección entre los referentes contenidos en la información previa: los hijos de Ana. Además como el nombre en chino es neutral a la oposición de número; con el nombre 孩子/hai zi/ "hijo" que se menciona con anterioridad, no se sabe si trata de uno o varios. Sin embargo, la repetición del mismo CLF.Num en chino en uso anafórico hace posible la lectura plural del nombre. Dicho de otra manera, aparte de la función anafórica, la repetición del mismo CLF.Num es un indicador de pluralidad.

El tercer instrumento que expresa la definitud es el posicional. La relación entre la definitud en chino y la posición u orden de palabras ha sido el tema de investigación de numerosos lingüistas; por ejemplo, Lü (1990); Sun y Givón (1985); Chen (2003); Li (1986) y Huang (1999). Basándose en los trabajos anteriores, Chen (2004: 1168) propone las siguientes posiciones en chino que tienden a tener lecturas definidas o indefinidas:

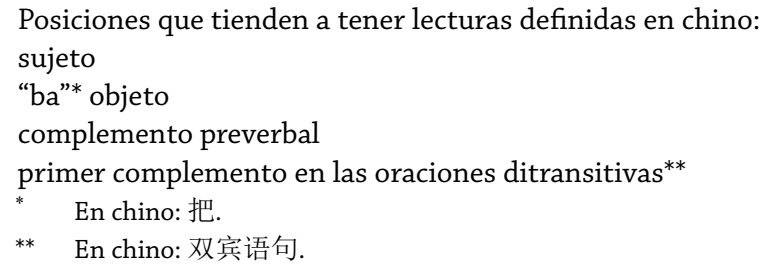


Posiciones que tienden a tener lecturas indefinidas en chino:

complemento del verbo presentativo "you"

SN posverbal en las oraciones presentativas

SN posverbal en las oraciones existenciales

segundo complemento en las oraciones ditransitivas

Hay que llamar la atención sobre la palabra "tienden" en (8) y (9), ya que el mismo autor ha citado en su artículo una larga lista de excepciones que no corresponden con las tendencias generales arriba expuestas.

Lin (2003) expone en su tesis doctoral dos criterios para indicar la definitud en chino: el de orden y el del tópico:

\begin{abstract}
Criterio de orden:
El orden de palabras en chino desempeña un papel importante para dar cuenta del rasgo definido o indefinido de los SSNN. El SN que se pospone al verbo tiende a ser un SN indefinido, y el que se antepone al verbo es definido.
\end{abstract}

Criterio de tópico:

En chino, el SN que desempeña el papel de tópico adquiere una interpretación definida o genérica.

Estos dos criterios arrojan cierta luz sobre el estudio de la definitud en chino. Sin embargo, es fácil encontrar excepciones que quedan fuera de los criterios arriba expuestos. Veamos el pequeño segmento que hemos citado:

他是个独自在湾流中的一条小船上钓鱼的老人, 至今已去了八十四

天, 一条鱼也没逮住。头四十天里, 有个男孩子(un muchacho)跟他在

一起。可是, [...]

老人教会了孩子* (a) (Ø muchacho)捕鱼，孩子(b) ( Ø muchacho)爱他。

* La traducción original es 这孩子 (zhe hai zi) (este muchacho). Con el propósito de facilitar la investigación, empleamos en este caso la forma cero $\varnothing$ según el principio de sustitución que establece la escala GH.

“Era un viejo que pescaba solo en un bote en la corriente del Golfo y hacía ochenta y cuatro días que no cogía un pez. En los primeros cuarenta días había tenido consigo a un muchacho.

Pero [...]

El viejo había enseñado al muchacho (a) a pescar, y el muchacho (b) le tenía cariño."

En (11a), el nombre 孩子/hai zi/ ( $\varnothing$ muchacho) está pospuesto al verbo, mientras que la misma palabra se encuentra antepuesta en (11b). Sin embargo, 
la diferencia posicional no altera de ninguna manera la definitud en estos casos. Ambos SSNN son definidos.

$$
\begin{aligned}
& \text { 他是个独自在湾流中的一条小船上钓鱼的老人, 至今已去了八十四 } \\
& \text { 天, 一条鱼也没逮住。头四十天里, 有个男孩子 (un muchacho) 跟 } \\
& \text { 他在一起。可是, 过了四十天还没捉到一条鱼, 孩子(a) ( }(\text { N) 的父 } \\
& \text { 母对他说, 老人如今准时十足地 “倒了血霉”, 这就是说, 倒霉到了 } \\
& \text { 极点, 于是孩子(b) ( }(\varnothing \mathrm{N}) \text { 听从了他们的吩咐, 上了另外一条船, 头 } \\
& \text { 一个礼拜就捕到了三条好鱼。 }
\end{aligned}
$$

"Era un viejo que pescaba solo en un bote en la corriente del Golfo y hacía ochenta y cuatro días que no cogía un pez. En los primeros cuarenta días había tenido consigo a un muchacho. Pero después de cuarenta días sin haber pescado, los padres del muchacho (a) le habían dicho que el viejo estaba definitiva y rematadamente salao lo cual era la peor forma de la mala suerte; y por orden de sus padres, el muchacho (b) había salido en otro bote, que cogió tres buenos peces la primera semana."

En (12), el primer 孩子/hai zi/ ( $\varnothing$ muchacho) no es tópico, mientras que el segundo constituye el tópico de la oración. No obstante, ambos son SSNN definidos, lo cual ha demostrado la falta de universalidad del criterio de tópico.

Creemos que la mayor debilidad de los numerosos trabajos acerca de la definitud en chino consiste en la falta de una visión textual. Los investigadores siempre trabajan con oraciones sueltas y nunca toman en consideración los diversos factores textuales y contextuales. Observamos que en todos los casos arriba expuestos, la definitud del nombre escueto孩子/hai zi/ ( $\varnothing$ muchacho) queda justificada con la presencia del sN一个男孩子/yi ge nan hai zi/ (un muchacho), que se encuentra al principio del texto. Por lo tanto, afirmamos que en cuanto a la definitud en chino, además de enunciados sueltos, debemos trabajar con textos.

\section{La definitud en chino a nivel textual}

Hemos señalado en el apartado anterior que es indispensable tener una visión textual para analizar la definitud en chino. Dicho de otra manera, aparte de los instrumentos léxicos, morfológicos y posicionales que nos ha descrito Chen (2004), debemos equiparnos además con el instrumento textual o discursivo. Con esta afirmación, complementemos el esquema de los instrumentos de definitud en chino propuesto por Chen (2004: 1151) en lo que sigue: 

a. léxico
b. morfológico
c. posicional
d. textual

De hecho, al explicar las excepciones que no corresponden con las tendencias que indican los instrumentos posicionales, el mismo Chen (2004: 1170) ha señalado la necesidad de un enfoque textual:

\footnotetext{
"Text counts may reveal a preference for one category of expressions rather than another."
}

Las mismas ideas las ha expresado Lyons (1999: 89) al analizar la relación de definitud con el orden de palabras en chino. Según este autor, en los casos ambiguos en cuanto a la lectura definida o indefinida, siempre es el contexto el que aclara la situación.

Queremos resaltar la importancia que implica adoptar una perspectiva textual para el chino. Como hemos explicado en los apartados anteriores, la mayoría de los SSNN definidos y anafóricos en chino son nombres escuetos, que no llevan consigo ninguna marca. En español, el artículo definido en la anáfora inicia y dirige todo el proceso interpretativo. En contraste con ello, en chino no se encuentra tal marca. El único remedio que nos queda es iniciar todo el proceso interpretativo a partir de la información contextual.

Retomemos el ejemplo del muchacho. Con el sintagma de CLF.Num 一个/ yi ge/ (uno + CLF. Num), el nombre男孩子/nan hai zi/ "muchacho" queda determinado. Al mismo tiempo, el SN一个男孩子/yi ge nan hai zi/ “un muchacho" recién determinado activa en el texto posterior todos los nombres que tengan relación correferencial 孩子/hai zi/ ( $\varnothing$ muchacho) u otros que tengan cierta asociación conceptual para que éstos puedan ser referenciales y definidos. Como muestra el esquema (13), la definitud de todos los $\varnothing \mathrm{N}$ у $\varnothing \mathrm{N}_{1}, \varnothing \mathrm{N}_{2}, \varnothing \mathrm{N}_{3}$... es garantizada con la presencia de su activador: un $\mathrm{N}$ o una expresión no nominal que pueden establecer con ellos una relación directa o indirecta. En este sentido, la definitud no tiene mucho que ver con la posición oracional.
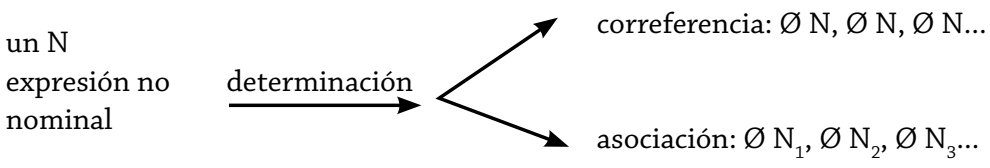
Veremos ahora el famoso ejemplo que suele ser citado para mostrar el contraste de definitud vs. indefinitud en chino:

a. 客人/ke ren/ 'visitante'来/lai/ 'venir'了。

"Vienen los visitantes."

b. 来/lai/ 'venir' 客人/ke ren/ 'visitante'了。

"Vienen unos visitantes."

Se suele afirmar que el nombre escueto preverbal客人/ke ren/ “visitante” que se encuentra en (14a) es definido, mientras que el mismo nombre posverbal en (14b) es indefinido. En términos generales, esta afirmación es correcta, puesto que si algo es inicial, entonces típicamente es definido. No obstante, si insertamos (14b) en un determinado contexto, el mismo nombre puede tener una lectura definida. Veamos un contexto que nos propone Diao (2007):

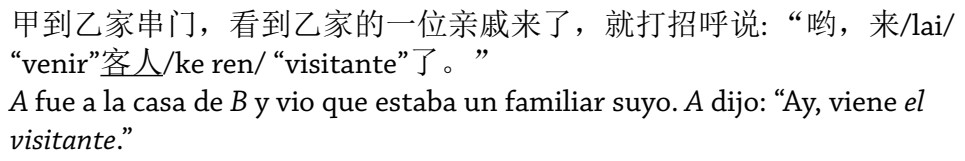

$\mathrm{Al}$ insertarse en el contexto (15), el nombre escueto 客人/ke ren/ “visitante” posverbal adquiere una lectura definida refiriéndose al familiar de $B$. Es decir, es el contexto el que decide la definitud. Por lo tanto, afirmamos que sin tomar en consideración los factores contextuales, se puede decir en términos generales que los nombres escuetos definidos suelen ocupar la posición preverbal, y los indefinidos, posverbal. Sin embargo, al insertarse en un contexto determinado, la lectura definida o indefinida será más afectada por el contexto que por la posición.

\section{Conclusión}

A modo de resumen, argumentamos que en español, con el artículo los conceptos definidos son marcados, mientras que en la lengua china, con los CLF. Num, lo que es representado gramaticalmente es la indefinitud. Como en chino no se encuentra la representación gramaticalizada de definitud, aparte de algunas indicaciones léxicas, morfológicas y posicionales, debemos adoptar una visión textual para determinar la lectura definida o indefinida de los 
grupos nominales. Muchos nombres escuetos en las posiciones que tienden a tener lecturas indefinidas adquieren definitud por ser anafóricos (directos o asociativos) o verse afectados por diversos factores textuales y contextuales.

\section{Referencias bibliográficas}

Alonso, Amado (1967), "Estilística y gramática del artículo en español", Estudios lingüísticos. Temas españoles, Madrid: Gredos.

Chen, Ping (2003), "Indefinite determiner introducing definite referent: A special use of 'one' + classifier in Chinese”, Lingua, núm. 113, pp. 1169-1184.

- (2004), "Identifiability and definiteness in Chinese", Linguistics, 42(6), pp. 1129-1184.

Diao, Shilan (2007), "The referencial properties of bare NP and their syntactic distribution", Journal of Hubei Normal University (Philosophy and Social Science), núm. 27, pp. 71-75.

Dong, Xiufang (2010), "Diachronic changes of the referential properties of bare nouns in Chinese", Studies in Language and Linguistics, 2010(1), pp. 15-24.

Heine, Bernd (1997), Cognitive Foundations of Grammar, Oxford: Oxford University Press.

Huang, Shuanfan (1999), "The emergence of a grammatical category definite article in spoken Chinese", Journal of Pragmatics, núm. 31, pp. 77-94.

Li, Lingding (1986), Sentence Patterns in Modern Chinese, Beijing: Shangwu Yinshuguan.

Lin, Tzu-Ju (2003), La adquisición y el uso del artículo por alumnos chinos, tesis doctoral, Alcalá: Universidad de Alcalá.

Lü, Shuxiang (1990), "Essentials of Chinese Grammar", Lü Shuxiang Wenji, Beijing: Shangwu Yinshuguan.

Lyons, Christopher (1999), Definiteness, Nueva York: Cambridge University Press.

RAE (2010), Nueva gramática de la lengua española: Manual, Madrid: Espasa.

Sun, Chaofen, y Givón, Talmy (1985), "On the so-called sov word order in Mandarin Chinese: A quantified text study and its implications", Language, 61(2), pp. 329-351.

Xu, Yulong (1992), Introducción a la lingüistica contrastiva, Shanghai: Shanghai Waiyu Jiaoyu Chubanshe. 
Zhang, Yisheng (2001), "De CLF.Num a auxiliar: Análisis del proceso de gramaticalización del CLF.Num GE", presentado en la conferencia organizada por la Universidad de Hong Kong, La gramática del chino moderno en el siglo XXI, febrero 1 de 2001, Hong Kong. ख? 